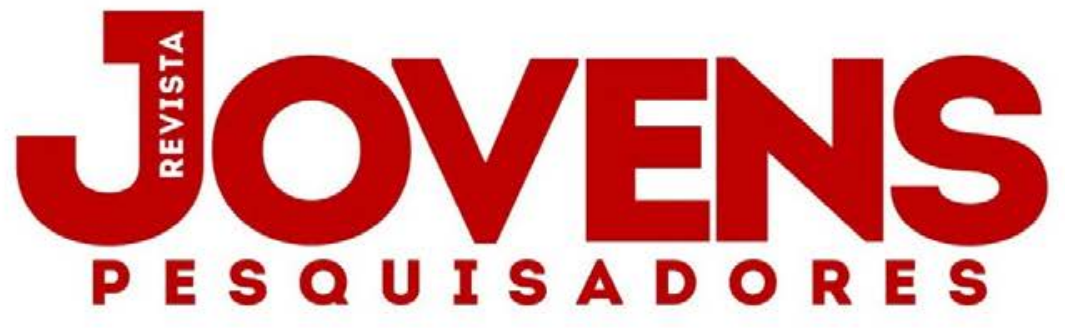

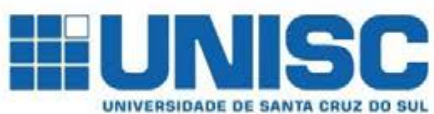

ISSN 2237-048X

Doi: $10.17058 /$ rjp.v8i2.11921

Recebido em: 04/04/2018

Aceito em: 22/06/2018

\title{
ASSOCIAÇÃO DE POLIMORFISMOS NOS GENES FTO, TMEM18E ADIPOQ, E CONCENTRAÇÃO SÉRICA DE ADIPONECTINA COM EXCESSO DE PESO E PERFIL BIOQUÍMICO EM ESCOLARES DE SANTA CRUZ DO SUL/RS - BRASIL
}

\author{
MATOS, W. L.1; RODRIGUES, S. T.2; KLINGER, E. I. ${ }^{\text {; }}$ REUTER, C. P. 4; TODENDI, P. F. 5; VALIM, A. R. M. ${ }^{6}$
}

PALAVRAS ChAVE: Obesidade. Escolares. TMEM18. FTO. ADIPOQ. Polimorfismo.

\section{RESUMO}

A obesidade é uma doença multifatorial e poligênica, relacionada ao desenvolvimento de complicações metabólicas e cardiorrespiratórias. Sugere-se que fatores genéticos contribuam de 40 a $70 \%$ na susceptibilidade de desenvolvimento da obesidade e, nesse contexto, Polimorfismos de Nucleotídeo Único (SNPs) nos genes FTO (Fat mass obesity-associated), TMEM18 (proteína transmembranar 18) e ADIPOQ (Adiponectina) se mostraram relacionados à alterações nos parâmetros antropométricos e bioquímicos. A partir disso, objetivou-se analisar a relação entre os SNPs nos genes FTO (rs9939609), TMEM18 (rs6548238) e ADIPOQ (rs182052), e adiponectina sérica com o excesso de peso e as características antropométricas e bioquímicas em escolares de Santa Cruz do Sul/RS. Esse estudo, de caráter transversal, contou com participação de 600 escolares, com idades entre 7 e 17 anos. A genotipagem dos SNPs foi realizada através de qPCR. 0 tratamento dos dados deu-se por estatística descritiva, ANOVA e Kruskal-Wallis. Observou-se neste estudo, que a prevalência de excesso de peso é de 38,3\%, que portadores do genótipo AT do rs9939609 (FTO) apresentaram maiores valores de índice de massa corporal $(p=0,042)$, percentual de gordura $(\% G)(p=0,045)$ e triglicerídeos $(p<0,001)$ e que portadores do genótipo CC, do rs6548238 (TMEM18) apresentaram maiores valores de \%G $(p=0,036)$. 0 SNP rs182052 (ADIPOQ) e a concentração sérica de adiponectina não se mostraram relacionados as variáveis estudadas.

\section{ASSOCIATION OF POLYMORPHISMS IN THE FTO, TMEM18 AND ADIPOQ GENES, AND SERUM ADIPONECTIN CONCENTRATION WITH EXCESS WEIGHT AND BIOCHEMICAL PROFILE IN SCHOOLCHILDREN FROM SANTA CRUZ DO SUL/RS - BRAZIL}

\author{
KEYWORDS: Obesity. Schoolchildren. TMEM18. FTO. ADIPOQ. Polymorphism.
}

\begin{abstract}
Obesity is a multifactorial and polygenic disease, related to the development of metabolic and cardiorespiratory complications. It's suggested that genetic factors contribute from 40 to $70 \%$ in the susceptibility of obesity development and, in this context, Single Nucleotide Polymorphisms (SNPs) in the FTO (Fat mass obesityassociated), TMEM18 (transmembrane protein 18) and ADIPOQ (Adiponectin) were related to changes in anthropometric and biochemical parameters. The aim of this study was to analyze the relationship between SNPs in the FTO (rs9939609), TMEM18 (rs6548238) and ADIPOQ (rs182052), and serum adiponectin with excess weight and anthropometric and biochemical characteristics in schoolchildren from Santa Cruz do Sul/RS. This cross-sectional study had the participation of 600 students, aged between 7 and 17 years. Genotyping of the SNPs was performed through qPCR. The treatment of the data was by descriptive statistic, ANOVA and KruskalWallis. In this study, the prevalence of overweight was 38.3\%, whereas those with the rs9939609 (FT0) AT genotype presented higher values of body mass index $(p=0.042)$, percentage of fat $(\% G)(p=0.045)$ and triglycerides $(p<0.001)$ and that patients with the CC genotype of rs6548238 (TMEM18) presented higher values of \%G $(p=0.036)$. The SNP rs182052 (ADIPOQ) and the serum concentration of adiponectin were not related to the studied variables.
\end{abstract}

\footnotetext{
1 Acadêmico do curso de Farmácia na Universidade de Santa Cruz do Sul.

2 Acadêmica do curso de Farmácia na Universidade de Santa Cruz do Sul.

${ }^{3}$ Graduada em Farmácia e Mestre em Promoção da Saúde na Universidade de Santa Cruz do Sul.

${ }^{4}$ Docente do Departamento de Educação Física e Saúde da Universidade de Santa Cruz do Sul.

${ }^{5}$ Doutoranda em Patologia pela Universidade Federal de Ciências da Saúde de Porto Alegre.

${ }^{6}$ Docente do Departamento de Biologia e Farmácia na Universidade de Santa Cruz do Sul.
} 


\section{INTRODUÇÃO}

A obesidade tem emergido como um dos maiores problemas de saúde pública em vários países do mundo, segundo um estudo da Global Burden of Disease (GBD) 2015 Obesity Collaborators. No Brasil, estudos de prevalência recentes, com crianças e adolescentes em idade escolar, demonstraram prevalência de excesso de peso de 27,0\% (BARBIAN et al., 2017) e 30,0\% (ANJOS; SILVEIRA, 2017).

Esses dados são particularmente preocupantes, uma vez que obesidade na infância tende a permanecer até a idade adulta (JUONALA et al., 2011), estando relacionada ao desenvolvimento de inúmeras comorbidades, dentre as quais: complicações metabólicas, psicossociais e respiratórias (USPSTF, 2005), doenças cardiovasculares, doença renal crônica, diabetes mellitus (SINGH et al., 2013), distúrbios musculoesqueléticos (JIANG et al., 2012) e alguns tipos de câncer (DONG et al., 2017; HIDAYAT et al., 2018). Além disso, o excesso de peso está associado ao risco duas vezes maior de desenvolver multimorbidades cardiometabólicas (KIVIMAKI et al., 2017).

A obesidade é compreendida como uma doença crônica multifatorial, e estudos sugerem que fatores genéticos contribuam de 40 a $70 \%$ na susceptibilidade de seu desenvolvimento (LOOS, 2009). Nesse sentido, Polimorfismos de Nucleotídeo Único (SNPs) nos genes FTO (Fat mass obesity-associated), TMEM18 (Proteína Transmembranar 18) e ADIPOQ (Adiponectina) têm se mostrado associados ao excesso de peso e alterações nos parâmetros antropométricos e bioquímicos (FRAYLING et al., 2007; WILLER, 2009; RICHARDSON et al., 2006), tornando-se alvos potenciais de pesquisas. Ademais, das proteínas codificadas pelos genes supracitados, níveis séricos baixos de adiponectina estão associados a excesso de adiposidade (KIM et al., 2016).

Com base nesses pressupostos, este estudo teve como objetivo investigar o efeito dos SNPs nos genes FTO (rs9939609), TMEM18 (rs6548238) e ADIPOQ (rs182052) relacionando-os com parâmetros clínicos e antropométricos em crianças e adolescentes de Santa Cruz do Sul/RS.

\section{REFERENCIAL TEÓRICO}

0 gene FTO possui 9 exons e 8 introns, localiza-se no cromossomo 16, na região cromossômica 16q12.2 (NCBI, 2017). Dentre os SNPs que estão associados ao excesso de peso, o rs9939609 é estudado com maior frequência (LUIS et al., 2012). Ele se encontra no intron 1 e é constituído pelos alelos A e T. Destes, o alelo A está diretamente associado a um maior acúmulo de gordura corporal, principalmente, quando se apresenta na forma homozigota (AA) (REUTER et al., 2016; LUIS et al., 2012). 0 estudo de Frayling et al. (2007), que envolveu 38.759 participantes, o primeiro a investigar essa relação, mostrou que $16 \%$ dos avaliados, que eram homozigotos para o alelo A, apresentaram cerca de $3 \mathrm{~kg}$ a mais quando comparados com aqueles que não herdaram o alelo de risco (FRAYLING et al., 2007). Posteriormente, o estudo de Yang et al. (2014), encontrou riscos de desenvolvimento de obesidade 1,47 e 3,32 vezes maiores para indivíduos heterozigotos (AT) e homozigotos (AA), respectivamente (YANG et al., 2014). A função deste gene ainda não foi completamente elucidada. Entretanto, encontrou-se uma alta expressão no núcleo arqueado do hipotálamo, cuja região tem papel importante no apetite (GERKEN et al., 2007; STRATIGOPOULOS et al., 2008).

0 gene $A D I P O Q$ localiza-se no cromossomo 3 na região cromossômica 3q27. Ele possui uma extensão de 16kb e contém 3 exons, que codificam a adiponectina, uma proteína plasmática com 244 aminoácidos, com $30 \mathrm{kDa}$ (HENNEMAN et al., 2010; NCBI, 2017). Secretada exclusivamente pelo tecido adiposo, a adiponectina está presente no plasma sanguíneo em grandes concentrações (KADOWAKI et al., 2006). Ela age regulando o metabolismo da glicose e lipídeos, aumentando a absorção da glicose e a oxidação da gordura no músculo esquelético e no fígado, além de diminuir a produção de glicose hepática (KHANDEKAR et al., 2011). Ademais, a adiponectina apresenta propriedades anti-inflamatórias e antiaterogênicas que estão associadas à obesidade (LEU et al., 2011), pois aumenta a síntese de citocinas, como a IL-10, diminui a produção de TNF- $\alpha$ e IL-6 e inibe a ativação do NF-Kß (KUKLA et al., 2011). Estudos demonstraram que os níveis individuais da adiponectina podem variar pela presença de determinados polimorfismos no gene ADIPOQ (HENNEMAN et al., 2010). 
0 gene TMEM18 está localizado no cromossomo 2, na região cromossômica 2 p25.3, sendo responsável pela codificação da proteína transmembranar 18 (NCBI, 2017). Descrito pela primeira vez em 2008, foi identificado como um gene TOP (do inglês: terminal oligo-pyrimidine), por apresentar uma sequência oligo-pirimidina na região terminal 5' do mRNA. (YAMASHITA et al., 2008). É expresso em níveis elevados no cérebro e particularmente no hipotálamo, região importante para os processos de regulação do peso (WILLER et al., 2009). Estudos apontam sua relação com obesidade na fase adulta e na infância (ÁLMEN et al., 2010; LIU et al., 2014). Existem, no mínimo, 46 SNPs independentes e nominalmente significativos do gene TMEM18 que estão associados a maior IMC e, dentre esses, os SNPs rs6548238, rs2867125 e rs7596758 são os mais significativos (LIU et al., 2014).

\section{MATERIAIS E MÉTODOS}

\subsection{DESENHO DO ESTUDO E AMOSTRA}

Estudo transversal composto por 600 crianças e adolescentes, com idades entre sete e 17 anos, de escolas da rede pública e privada de Santa Cruz do Sul/RS integrantes a pesquisa intitulada como "SAÚDE DOS ESCOLARES - FASE III" aprovada pelo CEP da Universidade de Santa Cruz do Sul (UNISC) sob protocolo $n^{\circ}$ 714.216/14. As coletas foram realizadas nos anos de 2014 e 2015 e as amostras utilizadas foram armazenadas em freezer a $80^{\circ} \mathrm{C}$. Este estudo atende os referenciais básicos da Bioética, previstos na Resolução 466/2012, através de assinatura, em duas vias, do Termo de Consentimento Livre e Esclarecido pelo pais ou responsáveis dos escolares convidados a participarem da pesquisa.

\subsection{MENSURAÇÕES CLÍNICAS}

Medidas antropométricas, tais como o IMC, Circunferência da Cintura (CC) e Percentual de Gordura (\%G) foram mensuradas. $O$ IMC foi classificado a partir das curvas de percentis da Organização Mundial da Saúde (WHO, 2007), de acordo com sexo e idade, considerando baixo peso (<p3), normal ( $\geq$ p3 e <p85), sobrepeso ( $\geq$ p85 e $<p 97)$ e obesidade ( $\geq$ p97). A CC foi avaliada através de fita métrica inelástica, utilizando como referência a parte mais estreita do tronco entre as costelas e a crista ilíaca e o quadril no nível do trocânter maior, sendo posteriormente classificada através dos critérios estabelecidos por Fernández et al. (2004), de acordo com sexo e idade, considerando circunferência normal percentil $<75$ e elevado com percentil $\geq 90$.

O \%G foi calculado através da equação de Slaugther et al. (1988), sendo posteriormente classificado de acordo com os dados de Lohman (1987) e separado em duas categorias: muito baixo/baixo/ótimo e moderadamente alto/alto/muito alto. As pressões arteriais sistólicas (PAS) e diastólicas (PAD) foram aferidas com o escolar em posição sentada, após repouso, e utilizou-se um esfigmomanômetro, com um estetoscópio no braço direito. Posteriormente, foram classificadas de acordo com os percentis de idade, gênero e altura considerando normal quando percentil $<90$ e risco percentil $\geq 90$ (SBC; SBH; SBN, 2010).

Foram coletados $10 \mathrm{~mL}$ de sangue por punção venosa, sendo $5 \mathrm{~mL}$ centrifugados para separação de soro utilizado para as análises bioquímicas e $5 \mathrm{~mL}$ anticoagulados com EDTA para a extração de DNA. Os exames bioquímicos foram realizados com kits comerciais Kovalent (Kovalent do Brasil Ltda), tendo sido medidos os níveis de glicose (GLI), colesterol total (CT), Colesterol LDL (LDL-C) e triglicerídeos (TG), sendo estes classificados em: normal e elevado. 0 Colesterol HDL (HDL-C) teve seus níveis classificados como: normal e baixo (NHLBI, 2012). 0 LDL-C foi calculado de acordo com a equação de Friedewald et al. (1972). A glicose foi considerada como normal quando <100 mg/dL e elevada quando variou de 100 a $125 \mathrm{mg} / \mathrm{dL}$ (ADA, 2011).

\subsection{ANÁLISE MOLECULAR DOS GENES FTO, ADIPOQE TMEM18}

Foram utilizados $500 \mu \mathrm{L}$ de cada amostra de sangue total (anticoagulado com EDTA) para a extração do DNA, através do método de Salting-out, conforme descrito por Miller, Dykes e Polesky (1988). Utilizando ensaios de discriminação alélica conforme instruções do fabricante, os genes foram analisados através da técnica de qPCR no equipamento StepOne Plus ${ }^{\circledR}$. Os ensaios FTO rs9939609 C_30090620_10, ADIPOQ rs182052 C__2412785_10, TMEM18 rs6548238 C__29311887_10 e Master Mix PCR Universal foram adquiridos da 
Applied Biosystems (Foster City, CA, USA). Essas análises foram realizadas no laboratório de Genética e Biotecnologia, Bloco 20, da UNISC.

\subsection{MENSURAÇÃO DA PROTEÍNA ADIPONECTINA}

A adiponectina foi mensurada por meio do teste ELISA, utilizando kit específico para determinação quantitativa in vitro. 0 Kit Adiponectin Human ELISA (Thermo Fisher Scientific, Waltham, MA USA) foi utilizado, seguindo as especificações do fabricante. Devido ao alto custo do kit parte da amostra foi selecionada de maneira aleatória $(n=154)$.

\subsection{MENSURAÇÃO DA PROTEÍNA ADIPONECTINA}

Os dados foram armazenados e tabulados em um banco de dados. As análises estatísticas foram realizadas utilizando o software SPSS 20.0. Uma análise descritiva através do teste qui-quadrado foi inicialmente utilizada. As distribuições normais $(\mathrm{HO})$ e as não-normais (H1) foram determinadas pelo teste não-paramétrico de Kolmogorov-Smirnov. 0 equilíbrio de Hardy-Weinberg (HWE) foi calculado comparando valores esperados e os observados, para todos os SNPs estudados. Para analisar a relação entre os genótipos de cada SNP com o IMC, CC, \%G e perfil lipídico e glicêmico foi realizado teste ANOVA (variáveis de distribuição normal) e Kruskal-Wallis (variáveis de distribuição não-normal).

\section{RESULTADOS E DISCUSSÃO}

A amostra, apresentada na Tabela 1, é composta majoritariamente por adolescentes $(82,8 \%)$ do sexo feminino (55,2\%), com mediana de idade de 12 anos (IIQ: 10,2-14,0). A cor/etnia (autodeclarada) mais prevalente na população avaliada foi branca $(70 \%)$, tendo em vista a origem caucasiana da região (FILHO; MONASTERIO, 2012), seguidos pela cor/etnia parda ou mulata $(19,7 \%)$ e negra $(9,0 \%)$. 
Tabela 1. Características sócio-demográficas e clínicas dos escolares

\begin{tabular}{|c|c|c|c|c|}
\hline \multirow{2}{*}{ Variáveis } & Total & Feminino & Masculino & \multirow{2}{*}{ Valor $p$} \\
\hline & $n=600(\%)$ & $n=331(\%)$ & $n=269(\%)$ & \\
\hline Idade (anos) & $12(10,2-14,0)$ & & & \\
\hline Faixa Etária & & & & 0,969 \\
\hline Criança (7 a 9 anos) & $103(17,2)$ & $57(17,2)$ & $46(17,1)$ & \\
\hline Adolescente (10 a 17 anos) & $497(82,8)$ & $274(82,8)$ & $223(82,9)$ & \\
\hline IMC $\left(\mathbf{k g} / \mathrm{m}^{2}\right)^{\mathrm{a}}$ & $20,4(18,2-23,2)$ & & & 0,735 \\
\hline Baixo peso/Normal & $370(61,7)$ & $207(55,9)$ & $163(44,1)$ & \\
\hline Sobrepeso & $123(20,5)$ & $64(19,3)$ & $59(21,9)$ & \\
\hline Obesidade & $107(17,8)$ & $60(18,1)$ & $47(17,5)$ & \\
\hline $\mathrm{CC}(\mathrm{cm})^{\mathrm{b}}$ & $66,0(60,4-72,6)$ & & & 0,171 \\
\hline Normal & $485(80,8)$ & $261(78,9)$ & $224(83,3)$ & \\
\hline Elevado & $115(19,2)$ & $70(21,1)$ & $45(16,7)$ & \\
\hline$\% G^{c}$ & $20,81 \pm 7,83$ & & & $0,001 *$ \\
\hline Muito baixo, baixo e excelente & $379(63,2)$ & $189(57,1)$ & $190(70,6)$ & \\
\hline $\begin{array}{l}\text { Moderadamente alto, alto e } \\
\text { muito alto }\end{array}$ & $221(36,8)$ & $142(42,9)$ & $79(29,4)$ & \\
\hline Colesterol Total (mg/dL)d & $165,13 \pm 28,95$ & & & 0,122 \\
\hline Normal & $345(57,5)$ & $181(54,7)$ & $164(61,0)$ & \\
\hline Elevado & $255(42,5)$ & $150(45,3)$ & $105(39,0)$ & \\
\hline HDL-C (mg/dL)e & $88,51 \pm 24,51$ & & & 0,364 \\
\hline Normal & $585(97,5)$ & $321(97,0)$ & $264(98,1)$ & \\
\hline Baixo & $15(2,5)$ & $10(3,0)$ & $5(1,9)$ & \\
\hline Triglicerídeos (mg/dL) & $62,1(46,2-88,9)$ & & & $0,001 *$ \\
\hline Normal & $449(74,8)$ & $230(69,5)$ & $219(81,4)$ & \\
\hline Elevado & $151(25,2)$ & $101(30,5)$ & $50(18,6)$ & \\
\hline LDL-C (mg/dL)g & $63,13 \pm 11,63$ & & & 0,465 \\
\hline Normal & $492(82,0)$ & $268(81,0)$ & $224(83,3)$ & \\
\hline Elevado & $108(18,0)$ & $63(19,0)$ & $45(16,7)$ & \\
\hline Glicose $(\mathbf{m g} / \mathrm{dL})^{\mathrm{h}}$ & $89,0(83,0-94,0)$ & & & 0,694 \\
\hline Normal & $564(94,0)$ & $310(93,7)$ & $254(94,4)$ & \\
\hline Elevada & $36(6,0)$ & $21(6,3)$ & $15(5,6)$ & \\
\hline PAS (mmHg)' & $100,0(95,0-120,0)$ & & & 0,235 \\
\hline Normal & $494(82,3)$ & $297(80,7)$ & $227(84,4)$ & \\
\hline Risco & $106(17,7)$ & $64(19,3)$ & $42(15,6)$ & \\
\hline PAD (mmHg) & $60,0(60,0-70,0)$ & & & 0,441 \\
\hline Normal & $510(85,0)$ & $278(84,0)$ & $232(86,2)$ & \\
\hline
\end{tabular}

a Índice de Massa Corporal (IMC): Baixo peso/normal ( $p<5$ e $p<85)$, sobrepeso ( $p \geq 85$ e $p<95)$ e obesidade ( $p \geq 95)$. ${ }^{b}$ Circunferência da Cintura (CC): normal $(p<75)$ e elevado ( $\left.p \geq 90\right)$. ${ }^{c}$ Percentual de Gordura (\%G): segundo Lohman

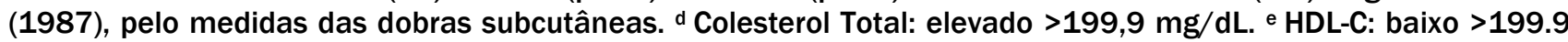

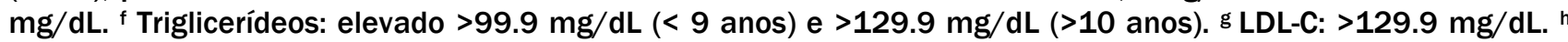
Glicose: elevada $>99,9 \mathrm{mg} / \mathrm{dL}$. ' Pressão Arterial Sistólica e Diastólica: normal $(p<90)$ e risco $(p \geq 90)$. Valor de $p$ : teste de qui-quadrado. *Valores significativos $(p<0,05)$ 
A prevalência de sobrepeso/obesidade da amostra estudada é de 38,3\%, observa-se também que 19,2\% e $36,8 \%$ dos sujeitos apresentam CC e \%G elevados, respectivamente. Dentre as variáveis clínicas analisadas o maior percentual foi de triglicerídeos, onde $25,2 \%$ dos sujeitos apresentaram valores alterados. Quando a amostra foi categorizada por sexo, observa-se que as meninas apresentaram \%G moderadamente alto, alto e muito alto $(42,9 \%)$, se comparado ao sexo oposto $(29,4 \%)(p=0,001)$. 0 mesmo se repete para os níveis séricos de TG, em que $30,5 \%$ das meninas foram classificados com TG elevado e nos meninos essa frequência foi $18,6 \%$ $(p=0,001)$.

A prevalência de sobrepeso e obesidade na população estudada (38,3\%), apresenta-se elevada se comparado a um estudo de âmbito nacional (ANJOS; SILVEIRA, 2017), com escolares de três a 17 anos, onde o excesso de massa corporal foi de $27,6 \%$ nas meninas e $32,3 \%$ nos meninos, representando $30,0 \%$ dos indivíduos no total. No presente estudo, a prevalência de excesso de peso também é mais elevada em meninos (39,4\%) do que em meninas (37,5\%). Além disso, observando-se dados de prevalência de excesso de peso em escolares, através de uma série de pesquisas realizadas na cidade de Santa Cruz do Sul/RS, nota-se um caráter crescente, onde a prevalência de excesso de peso em escolares na cidade nos anos de 2005, 2010 e 2016 eram de 20,6\% (REUTER et al., 2012), 26,7\% (BURGOS et al., 2010) e 34,5\% (REUTER et al., 2016), respectivamente, um crescimento sucessivo que se repete no presente estudo.

Os SNPs estudados estavam em equilíbrio de Hardy-Weinberg, apresentando valores semelhantes aos esperados, como demonstrado na Tabela 2, sendo os genótipos mais frequentes: AT para FTO rs9939609 (49,3\%), CC para TMEM18 rs6548238 (73,7\%) e AG para ADIPOQ rs182052 (44,8\%). A presença do alelo de risco do SNP rs9939609 (FTO) varia entre diferentes populações (GÁRCIA-SóLIS et al., 2016). No presente estudo, a frequência do alelo $A$ foi de $38,2 \%$. Em uma pesquisa anterior com a mesma população a frequência foi de 35,5\% (REUTER et al., 2016) e frequências de $18 \%$ e $10 \%$ foram encontradas em escolares mexicanos (GÁRCIA-SÓLIS et al., 2016) e em populações chinesas (LI et al., 2008), respectivamente. 0 alelo T do SNP rs6548238 (TMEM18) é o mais frequente, sendo encontrado em 85,5\% dos indivíduos, frequência semelhante a encontrada em escolares mexicanos (89,5\%) (GÁRCIA-SóLIS et al., 2016) e em crianças neozelandesas (84,4\%) (KRISHNAN et al., 2017). Já o alelo C do SNP rs182052 (ADIPOQ) foi observado em 63,5\% dos indivíduos, frequência similar aos 63,0\% encontrados em escolares mexicanos (MUNOZ-YANEZ et al., 2016).

Tabela 2. Frequência genotípica e alélica dos SNPs analisados

\begin{tabular}{|c|c|c|c|}
\hline & $\begin{array}{l}\text { Frequência observada } \\
\qquad n=600(\%)\end{array}$ & $\begin{array}{l}\text { Frequência esperada } \\
\qquad N=600(\%)\end{array}$ & $p$ \\
\hline FTO rs9939609 & & & 0,937 \\
\hline AA & $81(13,5)$ & $87(14,5)$ & \\
\hline AT & $296(49,3)$ & $283(47,2)$ & \\
\hline$\pi$ & $223(37,2)$ & $230(38,3)$ & \\
\hline $\mathrm{T}$ & $742(61,8)$ & & \\
\hline A & $458(38,2)$ & & \\
\hline TMEM18 rs6548238 & & & 0,815 \\
\hline$\pi$ & $16(2,7)$ & $13(2,1)$ & \\
\hline CT & $142(23,7)$ & $149(24,8)$ & \\
\hline $\mathrm{CC}$ & $442(73,7)$ & $439(73,1)$ & \\
\hline $\mathbf{T}$ & $1026(85,5)$ & & \\
\hline C & $174(14,5)$ & & \\
\hline ADIPOQ rs182052 & & & 0,965 \\
\hline AA & $84(14,0)$ & $79(13,2)$ & \\
\hline$A G$ & $269(44,8)$ & $279(46,5)$ & \\
\hline GG & $247(41,2)$ & $245(40,8)$ & \\
\hline G & $763(63,6)$ & & \\
\hline A & $437(36,4)$ & & \\
\hline
\end{tabular}


A relação entre os genótipos dos 3 SNPs estudados com os parâmetros antropométricos e clínicos, dispostos na Tabela 3 (A, B e C), mostram uma associação positiva entre genótipos do rs9939609 do FTO e uma elevação no IMC, no \%G e nos níveis séricos de TG. Foi observado que portadores do alelo A apresentaram maiores valores de IMC ( $p=0,042)$, com mediana de $20,48 \mathrm{~kg} / \mathrm{m}^{2}$ (IIQ: $17,99-23,27$ ) para homozigotos AA e $20,97 \mathrm{~kg} / \mathrm{m}^{2}$ (IIQ: 18,40-23,46) para heterozigotos AT, esse último, apresentou na mediana de IMC um acréscimo de $1,32 \mathrm{~kg} / \mathrm{m}^{2}$ se comparado aos indivíduos $T$. Já no \%G, nota-se que portadores do genótipo AT, com média de $21,61 \%$ $( \pm 7,96)$, apresentam cerca de $1,6 \%$ mais gordura corporal se comparado aos demais genótipos $(p=0,045)$.

A relação entre o alelo de risco (A) para o rs9939609 (FTO) e uma elevação do IMC já vem sendo relatada na literatura a mais de uma década (Frayling et al., 2007). Em estudo recente com a mesma população Reuter et al. (2016) demonstraram, em uma amostra de 406 escolares que homozigotos AA para esse SNP são mais frequentemente classificados com sobrepeso/obesidade $(57,4 \%)$ quando comparado às crianças com os genótipos AT (28,9\%) e TT (33,1\%). Por sua vez, Zhou et al. (2017) encontraram, em um estudo com 942 indivíduos de uma região da Austrália, com idades entre 6 meses e 25 anos, que portadores do genótipo AA possuem um acréscimo 1,38 kg/m² IMC (IC 95\%, 0,40-2,36, p=0,006) em comparação aos indivíduos TT, valor próximo ao encontrado no presente estudo.

Zhou et al. (2017) observaram que em comparação com o genótipo TT, o genótipo AA foi associado a um aumento no \%G de 3,15\% (IC 95\%, 1,27-5,04, p=0,001) semelhante ao observado no presente estudo. Além disso, observaram que indivíduos portadores no genótipo AT, apresentaram \% $0,83 \%$ superior ao genótipo $\mathrm{TT}$, resultado semelhante ao que foi encontrado no presente estudo, onde o genótipo AT foi relacionado a um valor aumentando nesse parâmetro. Entretanto, não foi observado, na população estudada, aumento de \%G em homozigotos AA, em comparação ao TT.

Uma associação positiva $(p=0,023)$ também foi encontrada entre os portadores do genótipo AT para o SNP rs9939609 (FTO) e um aumento nos níveis plasmáticos de TG. Portadores desse genótipo apresentaram mediana de $67,45 \mathrm{mg} / \mathrm{dL}$ (IIQ: 50,0-96,22) de TG sérico, aproximadamente $10 \mathrm{mg} / \mathrm{dL}$ a mais em comparação ao demais genótipos. Freathy et al. (2008), em uma meta-análise, também encontraram associação entre o genótipo do FTO rs9939609 e traços metabólicos, onde portadores do alelo de risco (A) apresentaram maiores níveis séricos de TG $(0,028 \mathrm{nmol} / \mathrm{L}$; IIQ: $0,003-0,052 ; p=0,025)$ se comparado com os não portadores. Essa associação também foi relatada por Tercjak-Řecko et al. (2012), em um estudo com crianças e adolescentes poloneses de quatro a 18 anos, mas apenas com relação ao genótipo $A A(p=0,04)$. Além disso, a presença do alelo de risco foi associada a um TG médio ajustado $0,1 \mathrm{mmol} / \mathrm{L}$ maior $(p=0,002)$ em comparação com homozigotos $\Pi$, em um estudo com 4.897 pacientes de uma região da Escócia (DONEY et al., 2009). No presente estudo, o genótipo AA não apresentou diferença significativa de TG em relação ao genótipo TT, o que não nos permite agregar esse aumento unicamente a presença do alelo $\mathrm{A}$, contrariando os estudos supracitados, onde o genótipo AA apresentou níveis mais elevados de TG sérico. 
Tabela 3. Comparação das médias e medianas dos dados antropométricos e clínicos com os SNPs analisados.

\begin{tabular}{|c|c|c|c|c|}
\hline \multirow[t]{2}{*}{ A) } & \multicolumn{3}{|c|}{$\begin{array}{c}\text { FTO rs9939609 } \\
\text { Média (士DP)* / Mediana (IIQ)* }\end{array}$} & \multirow[b]{2}{*}{$p$} \\
\hline & AAt & AT & $\pi$ & \\
\hline IMC $\left(\mathrm{Kg} / \mathrm{m}^{2}\right)^{\#}$ & $20,48(17,99-23,27)$ & $20,97(18,40-23,46)$ & $19,65(17,98-22,21)$ & $0,042 * *$ \\
\hline CC $(\mathrm{cm})^{\#}$ & $66,00(58,65-72,45)$ & $66,75(61,00-74,00)$ & $66,00(60,20-72,00)$ & 0,195 \\
\hline$\% G(\%)^{*}$ & $19,97 \pm 7,76$ & $21,61 \pm 7,96$ & $20,04 \pm 7,59$ & $0,045 * *$ \\
\hline PAS (mmHg)\# & $110,0(98,0-120,0)$ & $102,0(95,0-120,0)$ & $100,0(92,0-110,0)$ & 0,481 \\
\hline PAD (mmHg)* & $70,0(60,0-70,0)$ & $61,0(60,0-70,0)$ & $60,0(60,0-70,0)$ & 0,109 \\
\hline $\mathrm{HDL}(\mathrm{mg} / \mathrm{dL})^{*}$ & $62,86 \pm 12,31$ & $62,42 \pm 11,28$ & $64,17 \pm 11,80$ & 0,230 \\
\hline $\mathrm{LDL}(\mathrm{mg} / \mathrm{dL})^{*}$ & $84,54 \pm 26,56$ & $89,87 \pm 23,82$ & $86,87 \pm 24,54$ & 0,149 \\
\hline CT $(\mathrm{mg} / \mathrm{dL})^{*}$ & $162,88 \pm 32,06$ & $167,32 \pm 28,52$ & $163,05 \pm 28,25$ & 0,189 \\
\hline $\mathrm{TG}(\mathrm{mg} / \mathrm{dL})^{*}$ & $57,30(44,90-93,05)$ & $67,45(50,0-96,22)$ & $58,70(41,4-79,10)$ & $<0,001^{* *}$ \\
\hline Glicose $(\mathrm{mg} / \mathrm{dl})^{\#}$ & $89,00(83,00-94,00)$ & $89,00(83,00-94,00)$ & $89,00(84,00-95,00)$ & 0,874 \\
\hline \multirow[t]{2}{*}{ B) } & \multicolumn{3}{|c|}{$\begin{array}{c}\text { TMEM18 rs6548238 } \\
\text { Média ( }(\mathrm{DP})^{*} / \text { Mediana (IIQ)* }\end{array}$} & \\
\hline & $\pi+$ & Ст & $\mathrm{CC}$ & $\boldsymbol{P}$ \\
\hline IMC $\left(\mathrm{Kg} / \mathbf{m}^{2}\right)^{\#}$ & $19,14(16,43-21,04)$ & $19,67(17,80-22,00)$ & $20,61(18,35-23,50)$ & 0,163 \\
\hline $\mathrm{cc}(\mathrm{cm})^{\#}$ & $60,00(56,00-71,55)$ & $64,70(60,10-72,00)$ & $67,00(61,00-73,00)$ & 0,091 \\
\hline$\% G(\%)^{*}$ & $17,86 \pm 5,50$ & $19,70 \pm 7,11$ & $21,27 \pm 8,07$ & 0,036 ** \\
\hline PAS (mmHg)" & $100,0(90,0-106,0)$ & $100,0(92,0-110,0)$ & $102,0(95,0-120,0)$ & 0,482 \\
\hline PAD (mmHg)" & $63,0(50,0-70,0)$ & $65,0(60,0-70,0)$ & $60,0(60,0-70,0)$ & 0,225 \\
\hline $\mathrm{HDL}(\mathrm{mg} / \mathrm{dL})^{*}$ & $64,80 \pm 6,60$ & $62,49 \pm 10,68$ & $63,28 \pm 12,06$ & 0,658 \\
\hline $\operatorname{LDL}(\mathrm{mg} / \mathrm{dL})^{*}$ & $90,66 \pm 18,73$ & $85,93 \pm 25,99$ & $88,61 \pm 24,21$ & 0,479 \\
\hline CT $(\mathrm{mg} / \mathrm{dL})^{*}$ & $167,56 \pm 19,06$ & $161,76 \pm 29,19$ & $166,13 \pm 29,14$ & 0,278 \\
\hline $\mathrm{TG}(\mathrm{mg} / \mathrm{dL})^{*}$ & $57,35(40,20-79,95)$ & $61,60(46,00-81,70)$ & $62,65(46,70-91,50)$ & 0,482 \\
\hline Glicose (mg/dl)\# & $100,0(90,0-106,0)$ & $100,0(92,0-110,0)$ & $102,0(95,0-120,0)$ & 0,592 \\
\hline
\end{tabular}




\begin{tabular}{ccccc}
\hline C) & \multicolumn{4}{c}{$\begin{array}{c}\text { ADIPOQ rs182052 } \\
\text { Média }( \pm D P)^{*} / \text { Mediana }(I I Q)^{*}\end{array}$} \\
\hline IMC (Kg/m²)* & AAt & AG & GG & $p$ \\
CC (cm)* & $21,02(18,20-23,68)$ & $20,40(18,17-23,16)$ & $20,25(18,14-22,94)$ & 0,535 \\
$\% G(\%)^{*}$ & $66,65(60,70-76,05)$ & $66,00(61,00-73,00)$ & $65,40(60,35-71,65)$ & 0,457 \\
PAS (mmHg)* & $21,49 \pm 7,81$ & $20,62 \pm 7,92$ & $20,77 \pm 7,76$ & 0,647 \\
PAD (mmHg)* & $102,0(97,5-120,0)$ & $102,0(98,0-120,0)$ & $100,0(90,0-110,0)$ & 0,828 \\
HDL (mg/dL)* & $61,0(60,0-70,0)$ & $60,0(60,0-70,0)$ & $60,0(60,0-70,0)$ & 0,921 \\
LDL (mg/dL)* & $63,05 \pm 11,23$ & $62,91 \pm 11,35$ & $63,40 \pm 12,09$ & 0,889 \\
CT (mg/dL)* & $87,00 \pm 22,45$ & $86,83 \pm 24,55$ & $89,70 \pm 25,12$ & 0,379 \\
TG (mg/dL)* & $165,36 \pm 27,36$ & $164,19 \pm 29,28$ & $166,09 \pm 29,20$ & 0,756 \\
Glicose (mg/dl)* & $70,10(46,50-96,80)$ & $61,90(44,50-89,30)$ & $61,00(47,65-83,10)$ & 0,173 \\
\hline
\end{tabular}

A - FTO rs9939609; B - TMEM18 rs6548238; C - ADIPOQ rs182052.

IMC, índice de massa corporal; CC, circunferência da cintura; \%G, percentual de gordura; PAS, pressão arterial sistólica; PAD, pressão arterial diastólica;

†Genótipo de risco segundo a literatura cientifica consultada. *Valores expressos em média e desvio padrão, distribuição normal, teste ANOVA. \#Valores expressos em mediana e intervalo interquartil, distribuição nãonormal, teste Kruskal-Wallis. **Valores estatisticamente significativos $(p<0,05)$.

0 alelo T do SNP rs6548238 (TMEM18) mostrou-se relacionado ao aumento de IMC (SRIVASTAVA et al., 2016) e é descrito como alelo de risco em algumas populações. Em contrapartida, no presente estudo, o genótipo CC se mostrou associado a uma elevação no \%G $(p=0,036)$. Observou-se que a presença do alelo $C$ conferiu um acréscimo de 3,41 e 1,84 às médias de $\% G$ dos genótipos CC $(21,27 \pm 8,07)$ e CT $(19,70 \pm 7,11)$, respetivamente, em comparação ao genótipo $\Pi(17,86 \pm 5,50)$. Essa relação também foi encontrada por Haupt et al. (2010), em um estudo com 1.469 indivíduos de ascendência alemã (>98\%), onde portadores do alelo $C$ apresentaram maiores valores para $\% G(p=0,02), 31,3 \%( \pm 0,3)$ e $30,0 \%( \pm 0,5)$ para CC e CT, respectivamente, se comparada com indivíduos $T(29,6 \% \pm 1,5)$. Krishnan et al. (2017), por sua vez, em um estudo de corte que acompanhou 1961 crianças neozelandesas (maioria de ascendência europeia), encontraram que o alelo T funciona como um alelo protetor, reduzindo o \% em 1,27\% (IIQ: 0,361-2,18) para heterozigotos CT e 2,65\% (IIQ: 0,210-5,10) para homozigotos TT, em comparação ao genótipo $\mathrm{CC}(p=0,0063$ e $p=0,0333$, respectivamente).

Posto isso, pode-se sugerir que o comportamento do alelo C do SNP rs6548238 (TMEM18) como alelo de risco para \%G, pode estar relacionado às características étnicas da população avaliada, sendo esta de ascendência europeia (FILHO; MONASTERIO, 2012), bem como nos estudos de Haupt et al. (2010) e Krishnan et al. (2017), com populações majoritariamente caucasianas. Com relação ao rs182052 do gene ADIPOQ, não se encontrou associação significativa entre o SNP e as variáveis avaliadas.

Avaliando a relação entre a proteína adiponectina e os parâmetros antropométricos, clínicos e bioquímicos, não se observou relação estatisticamente significativa na população avaliada. Nota-se, entretanto, que indivíduos categorizados com níveis normais de HDL-C, apresentam maiores concentrações séricas de adiponectina $(19,78$ $\mathrm{mg} / \mathrm{dL} \pm 10,00)$, se comparado aos limítrofes $(13,10 \mathrm{mg} / \mathrm{dL} \pm 7,81)$.

\section{CONCLUSÃO}

Com os dados apresentados, é possível observar através desse estudo que a prevalência de sobrepeso/obesidade nos escolares avaliados é de 38,3\%, que portadores do genótipo AT do SNP rs9939609 no gene FTO apresentam maior IMC e percentual de gordura, bem como níveis séricos elevados de triglicerídeos. 
Além disso, observou-se que portadores do genótipo CC, do rs6548238 no gene TMEM18 apresentaram maiores valores de \%G. Com relação ao SNP rs182052 (ADIPOQ) e a adiponectina sérica, não se encontrou associação significativa com as variáveis avaliadas nesse estudo.

\section{AGRADECIMENTOS}

Agradecemos à Universidade de Santa Cruz do Sul - UNISC, a Fundação de Amparo à Pesquisa do Estado do Rio Grande do Sul (FAPERGS), ao Programa de Pós-Graduação em Promoção da Saúde (PPGPS/UNISC) e a professora Miria Suzana Burgos. Os autores também agradecem aos escolares, pais e professores e aos voluntários por participarem da pesquisa.

\section{REFERÊNCIAS}

ALMÉN, M. S. et al. The obesity gene, TMEM18, is of ancient origin, found in majority of neuronal cells in all major brain regions and associated with obesity in severely obese children. BMC Med Genet, London, v. 11, n. 58, abr. 2010.

AMERICAN DIABETES ASSOCIATION (ADA). Diagnosis and classification of diabetes mellitus. Diabetes Care, Arlington, v. 34, p. 62-9, 2011.

ANJOS, L. A. dos; SILVEIRA, W. D. B. da. Nutritional status of schoolchildren of the National Child and Youth Education Teaching Network of the Social Service of Commerce (Sesc), Brazil, 2012. Ciênc. saúde coletiva, Rio de Janeiro, v. 22, n. 5, p. 1725-1734, Maio 2017.

AYINA, C. N. A.; NOUBIAP, J. J. N.; ETOUNDI NGOA, L. S. et al. Association of serum leptin and adiponectin with

anthropomorphic indices of obesity, blood lipids and insulin resistance in a Sub-Saharan African population. Lipids Health Dis., London, v. 15, n. 96, 2016.

BARBIAN, C. et al. Comparação do perfil nutricional, lipídico e glicêmico de crianças e adolescentes de diferentes hemisférios da zona rural de Santa Cruz do Sul - RS. CINERGIS, Santa Cruz do Sul, v. 18, n. 2, p. 01-06, Abr./Jun. 2017.

BURGOS, M. S. et al. Uma Análise entre Índices Pressóricos, Obesidade e Capacidade Cardiorrespiratória em Escolares. Arq Bras Cardiol., São Paulo, v. 94, n. 6, p. 788-793, 2010.

DONEY, A. S. F. et al. The FTO Gene is Associated with an Atherogenic Lipid Profile and Myocardial Infarction in Patients with Type 2 Diabetes: A Go-DARTS study. Circ. Cardiovasc. Genet., Dallas, v. 2, n. 3, p. 255-259, 2009.

DONG, Y. et al. Abdominal obesity and colorectal cancer risk: systematic review and meta-analysis of prospective studies. Biosci. Rep., London, v. 37, n. 6, p. 1-12, Dez. 2017.

FERNÁNDEZ, J. R. et al. Waist circumference percentiles in nationally representative samples of African-American, EuropeanAmerican, and Mexican-American children and adolescents. The Journal of Pediatrics, Cincinnati, v. 145, n. 4, p. 439-444, 2004.

FILHO, I. de C.; MONASTERIO, L. Immigration and the origins of regional inequality: Government-sponsored European migration to southern Brazil before World War I. Regional Science and Urban Economics, Amsterdã, v. 42, p. 794-807, 2012.

FRAYLING, T. M. et al. A Common Variant in the FTO Gene Is Associated with Body Mass Index and Predisposes to Childhood and Adult Obesity. Science, Washington DC, v. 316, n. 5826, p. 889-894, maio 2007.

FREATHY, R. M. et al. Common Variation in the FTO Gene Alters Diabetes-Related Metabolic Traits to the Extent Expected Given Its Effect on BMI. Diabetes, Arlington, v. 57, n. 5, p. 1419-142, 2008.

FRIEDEWALD W. T., LEVY R. I., FREDRICKSON D. S. Estimation of the concentration of low-density lipoprotein cholesterol in plasma, without use of the preparative ultracentrifuge. Clin. Chem., v. 18, p. 499-502, 1972.

GARCíA-SOLís, P. et al. Fat mass obesity-associated (FTO) (rs9939609) and melanocortin 4 receptor (MC4R) (rs17782313) SNP are positively associated with obesity and blood pressure in Mexican school-aged children. British Journal of Nutrition, Cambridge, v. 10, p. 1-7, 2016.

GBD 2015 OBESITY COLLABORATORS. Health Effects of Overweight and Obesity in 195 Countries over 25 Year. The New England Journal of Medicine, Waltham, v. 377, n. 1, p. 13-27, Jul. 2017.

GERKEN, T. et al. The obesity-associated FTO gene encodes a 2-oxoglutarate-dependent nucleic acid demethylase. Science, Washington DC, v. 318, n. 5855, p.1469-72, 2007.

HAUPT, A. et al. Novel Obesity Risk Loci Do Not Determine Distribution of Body Fat Depots: A Whole-body MRI/MRS study. Obesity, Silver Spring, v. 18, n. 6, p. 1212-7, 2010.

HENNEMAN, P. et al. Genetic architecture of plasma adiponectin overlaps with the genetics of metabolic syndrome-related traits. Diabetes Care, Arlington, v. 33, n. 4, p. 908-13, abr. 2010.

HIDAYAT, K.; YANG, C. M.; SHI, B. M. Body fatness at a young age, body fatness gain and risk of breast cancer: systematic review and meta-analysis of cohort studies. Obesity Reviews, London, v. 19, n. 2, p. 254-268, 2018.

JIANG, L. et al. Body mass index and susceptibility to knee osteoarthritis: a systematic review and meta-analysis. Joint Bone Spine, v. 79, n. 3, p. 291-7, 2012.

JUONALA, M. et al. Childhood Adiposity, Adult Adiposity, and Cardiovascular Risk Factors. The New England Journal of Medicine, Waltham, v. 365, p. 1876-1885, nov. 2011.

KADOWAKI, T. et al. Adiponectin and adiponectin receptors in insulin resistance, diabetes, and the metabolic syndrome. Endocrine Reviews, Washington DC, v. 116, n. 7, p. 439-450, 2006.

KHANDEKAR, M. J.; COHEN, P.; \& SPIEGELMAN, B. M. Molecular mechanisms of cancer development in obesity. Nature Reviews Cancer, London, v. 11, n. 12, p. 886-95, 2011.

KIM, J. Y. et al. Prospective Study of Serum Adiponectin and Incident Metabolic Syndrome. Diabetes Care, Arlington, v. 36, n. 6, p. 1547-1553, Jun. 2013. 
KIVIMÄKI, M. et al. Overweight, obesity, and risk of cardiometabolic multimorbidity: pooled analysis of individual-level data for 120813 adults from 16 cohort studies from the USA and Europe. The Lancet, London, v. 2, p. 277-85, Jun. 2017.

KRISHNAN, M. et al. Analysis of association of gene variants with obesity traits in New Zealand European children at 6 years of age. Molecular BioSystems, Washington DC, v. 13, n. 8, p. 1524-1533, 2017.

KUKLA, M., MAZUR, W., BUŁDAK, R. J., \& ZWIRSKA-KORCZALA, K. Potential role of leptin, adiponectin and three novel adipokines-visfatin, chemerin and vaspin-in chronic hepatitis. Molecular Medicine, Cambridge, v. 17, n. 11-12, p. 1397-410, 2011.

LEU, H. B. et al. Adiponectin gene polymorphism is selectively associated with the concomitant presence of metabolic syndrome and essential hypertension. PloS One, San Francisco, v. 6, n. 5, p. 1-8, 2011.

LI H., et al. Variants in the fat mass- and obesity-associated (FTO) gene are not associated with obesity in a Chinese Han population. Diabetes, Arlington, v. 57, p. 264-268, 2008.

LIU, C. T. et al. Sequence Variation in TMEM18 in Association with Body Mass Index: The Cohorts for Heart and Aging Research in Genomic Epidemiology (CHARGE) Targeted Sequencing Study. Circ Cardiovasc Genet, Dallas, v. 7, n. 3, p. 344-349, jun. 2014.

LOHMAN, Timothy. The use of skinfold to estimate body fatness on children and youth. Journal of Physical Education, Recreation e Dance, London, v. 58, n. 9, p. 98-102, 1987.

LOOS, R. J. F. Recent progress in the genetics of common obesity. British Journal of Clinical Pharmacology, Schenectady, v. 68, n. 6, p. 811-29, 2009.

LUIS, D. A. de et al. Relation of the Rs9939609 Gene Variant in FTO With Cardiovascular Risk Factor and Serum Adipokine Levels in Morbid Obese Patients. Nutr Hosp, Madrid, v. 27, n. 4, p. 1184-1189, Jul-Aug 2012.

MILLER, S. A.; DYKES, D. D.; POLESKY, H. F. A simple salting out procedure for extracting DNA from human nucleated cells. Nucleic Acids Research, Oxford, v. 16, n. 3, p. 1215, 1988.

MUÑOZ-YÁÑEZ, C. et al. Polymorphisms FTO rs9939609, PPARG rs1801282 and ADIPOQ rs4632532 and rs182052 but not lifestyle are associated with obesity related-traits in Mexican children. Genetics and Molecular Biology, v. 39, n. 4, p. 547-553, 2016.

National Center for Biotechnology Information - NCBI. ADIPOQ. 2017. Disponível em:

https://www.ncbi.nIm.nih.gov/gene/79068. Acesso em: 14 fev. 2017.

National Center for Biotechnology Information - NCBI. Fat mass and obesity associated. 2017. Disponível em:

https://www.ncbi.nIm.nih.gov/gene/79068. Acesso em: 14 Fev. 2017.

National Center for Biotechnology Information - NCBI. TMEM18. 2016. Disponível em:

https://www.ncbi.nlm.nih.gov/gene/129787. Acesso em: 14 fev. 2017.

NATIONAL HEART, LUNG, AND BLOOD INSTITUTE (NHLBI). NHLBI Expert panel on integrated guidelines for cardiovascular health and risk reduction in children and adolescents. NIH Publication. 2012.

REUTER, C. P. et al. Association between overweight and obesity in schoolchildren with rs9939609 polymorphism (FTO) and family history for obesity. J. Pediatr. (Rio J.), Porto Alegre, v. 92, n. 5, p. 493-498, Oct. 2016.

REUTER, É. M. et al. Obesity and arterial hypertension in schoolchildren from Santa Cruz do Sul - RS, Brazil. Rev. Assoc. Med. Bras., São Paulo, v. 58, n. 6, p. 666-672, Dec. 2012.

RICHARDSON, D. K. et al. Association between variants in the genes for adiponectin and its receptors with insulin resistance syndrome (IRS)-related phenotypes in Mexican Americans. Diabetologia, Bristol, v. 49, p. 2317-2328, 2006.

SINGH, G. M. et al. The age-specific quantitative effects of metabolic risk factors on cardiovascular diseases and diabetes: a pooled analysis. PloS One, San Francisco, v. 8, n. 7, p. e65174, 2013.

SLAUGTHER, M. H. et al. Skinfold equations for estimation of body fatness in children and youth. Human Biology, Washington, v. 60, n. 5, p. 709-723, 1988.

SOCIEDADE BRASILEIRA DE CARDIOLOGIA (SBC), SOCIEDADE BRASILEIRA DE HIPERTENSÃO (SBH), SOCIEDADE BRASILEIRA DE NEFROLOGIA (SBN). VI Diretrizes Brasileiras de Hipertensão. Arq Bras Cardiol., São Paulo, v. 95, n. 1 supl.1, p. 1-51, 2010. SRIVASTAVA, A. et al. A multianalytical approach to evaluate the association of 55 SNPs in 28 genes with obesity risk in North Indian adults. American Journal of Human Biology, Medford, v. 20, n. 2, p. 1-17, 2016.

STRATIGOPOULOS, G. et al. Regulation of FTO/Ftm gene expression in mice and humans. American journal of physiology Regulatory, integrative and comparative physiology, Bethesda, v. 294, n. 4, 2008.

TERCJAK-REĆKO, M. et al. Polymorphism rs9939609 of FTO gene is related to the Body Mass Index in children from podlaskie province. Med Wieku Rozwoj, Warszawa, v. 16, n. 1, p. 53-61, 2012.

USPSTF - US Preventive Service Task Force. Screening and interventions for overweight inchildren and adolescents: recommendation statement. Pediatrics, Philadelphia, v. 116, n. 1, p. 205-209, 2005.

WILLER, C. J. et al. Six new loci associated with body mass index highlight a neuronal influence on body weight regulation. Nat. Genet., New York, v. 41, p. 25-34, 2009.

WHO. World Health Organization. Growth reference data for 5-19 years. 2007. Disponivel em:

http://www.who.int/growthref/en/. Acesso em: 21 Jun. 2017.

YAMASHITA, R. et al. Comprehensive detection of human terminal oligo-pyrimidine (TOP) genes and analysis of their characteristics. Nucleic Acids Res, v. 36, p. 3707-3715, 2008.

YANG, M. et al. The Effects of Genetic Variation in FTO rs9939609 on Obesity and Dietary Preferences in Chinese Han Children and Adolescents. PloS One, San Francisco, v. 9, n. 8, aug. 2014.

ZHOU, Y. et al. rs9939609 FTO genotype associations with FTO methylation level influences body mass and telomere length in an Australian rural population. Int J Obes (Lond), London, v. 41, n. 9, p. 1427-1433, Set. 2017. 